Abanico Veterinario. Enero-Diciembre 2020; 10:1-8. http://dx.doi.org/10.21929/abavet2020.17

Artículo Original. Recibido: 01/02/2020. Aceptado: 03/07/2020. Publicado: 14/08/2020.

\title{
Acetato de deslorelina y gonadotropina coriónica humana y su respuesta ovulatoria en yeguas postparto
}

\author{
Deslorelin acetate and human corionic gonadotropine and its ovulatory response in \\ postpartum mares
}

\section{Chávez-Smith Emma ${ }^{1 \text { ID }}$, Gutiérrez-Arenas Diana ${ }^{1 \mathrm{ID}}$, Lechuga-Arana Arianna ${ }^{1 \mathrm{ID}}$, Avila-Ramos Fidel ${ }^{1}$ ID, Cadena-Villegas Said ${ }^{2}$ ID , Hernández-Marín Antonio*1 ID}

1'Departamento de Veterinaria y Zootecnia, División de Ciencias de la Vida, Campus Irapuato-Salamanca, Universidad de Guanajuato, km 9 carretera Irapuato-Silao, Exhacienda El Copal, Irapuato, Guanajuato. 36824. ${ }^{2}$ Colegio de Postgraduados, Campus Tabasco. Periférico Carlos A. Molina, km 3 carretera Cárdenas-Huimanguillo, Tabasco, México. 86500. *Autor de correspondencia y responsable de la investigación: José Antonio Hernández Marín. Departamento de Veterinaria y Zootecnia. División de Ciencias de la Vida. Campus Irapuato-Salamanca. Universidad de Guanajuato. ExHacienda el Copal km 9, carretera Irapuato-Silao, Irapuato, Guanajuato, México. C.P. 36824. jahmarin@ugto.mx, smithecha@gmail.com, diana.gutierrez@ugto.mx, alma_arianna@yahoo.com.mx, ledifar@ugto.mx, scadena@colpos.mx

\section{RESUMEN}

Con la finalidad de evaluar la tasa de crecimiento folicular, el tiempo de respuesta a la ovulación y el porcentaje de ovulación en yeguas postparto Cuarto de Milla se aplicaron dos tratamientos $(T)$, el primero con acetato de deslorelina (AD) y el segundo con gonadotropina coriónica humana (hCG). El estudio se realizó durante enero a junio de 2018, con dieciséis yeguas de 7 años, $450 \mathrm{~kg}$ de peso y 4.5 partos en promedio. El diámetro folicular ovárico se evaluó mediante ultrasonografía transrectal a tiempo real en respuesta a los tratamientos experimentales, en yeguas con folículos ováricos $\geq 35 \mathrm{~mm}$ de diámetro: T1 $(n=8)$ : Aplicación de $1 \mathrm{mg}$ de AD vía intramuscular (IM), y T2 $(n=8)$ : Aplicación de 2500 UI de hCG vía IM. No hubo diferencias $(P>0.05)$ en la tasa de crecimiento folicular (AD: $0.48 \pm 0.006 \mathrm{~mm}$ y hCG: $0.45 \pm 0.035$ $\mathrm{mm}$ ), el tiempo de respuesta a la ovulación (AD: $46.75 \pm 0.48 \mathrm{~h}$ y hCG: $56.00 \pm 8.00 \mathrm{~h}$ ) y el porcentaje de ovulación (AD: $8 / 8,100 \%$ y hCG: $7 / 8,87.5 \%$ ) en las yeguas postparto evaluadas. Se concluye que los tratamientos hormonales con acetato de deslorelina y la gonadotropina coriónica humana responden en la actividad ovárica en yeguas postparto Cuarto de Milla con folículos ováricos $\geq 35 \mathrm{~mm}$ de diámetro.

Palabras clave: Análogo de $\mathrm{GnRH}, \mathrm{hCG}$, reproducción en equinos, ovulación, foliculogénesis.

\begin{abstract}
In order to evaluate the rate of follicular growth, the response time to ovulation and the percentage of ovulation in Quarter Mile postpartum mares, two treatments $(T)$ were applied, the first with deslorelin acetate (DA) and the second with human chorionic gonadotropin (hCG). The study was conducted during January to June 2018, with sixteen 7-year-old mares, $450 \mathrm{~kg}$ of weight and 4.5 deliveries on average. Ovarian follicular diameter was evaluated by real-time transrectal ultrasonography in response to experimental treatments, in mares with ovarian follicles $\geq 35 \mathrm{~mm}$ in diameter: $T 1(n=8)$ : Application of $1 \mathrm{mg}$ of DA intramuscularly (IM), and T2 $(n=8)$ : Application of $2500 \mathrm{IU}$ of hCG via IM. There were no differences $(P>$ 0.05 ) in the rate of follicular growth (AD: $0.48 \pm 0.006 \mathrm{~mm}$ and hCG: $0.45 \pm 0.035 \mathrm{~mm}$ ), the time of response to ovulation (AD: $46.75 \pm 0.48 \mathrm{~h}$ and hCG: $56.00 \pm 8.00 \mathrm{~h}$ ) and the percentage of ovulation (AD: 8/8, 100\% and hCG: $7 / 8,87.5 \%$ ) in the postpartum mares evaluated. It is concluded that deslorelin acetate and human chorionic gonadotropin respond to ovarian activity in Quarter Mile postpartum mares with ovarian follicles $\geq$ $35 \mathrm{~mm}$ in diameter.
\end{abstract}

Keywords: $\mathrm{GnRH}$ analogue, hCG, equine reproduction, ovulation, folliculogenesis. 


\section{INTRODUCCIÓN}

La reproducción es considerada una de las fases más importantes en la vida de las yeguas, principalmente en aquellas que su fin zootécnico es ser reproductoras (CortésVidauri et al., 2018). Dentro de la población gonadotropa, las células productoras de gonadotropinas localizadas en el par distal y en el par tuberalis de la hipófisis y la heterogeneidad en el patrón de almacenamiento de la hormona luteinizante (LH) y de la hormona foliculoestimulante (FSH), se considera la base para la regulación diferencial de la secreción de gonadotropinas durante el ciclo reproductivo (Aurich, 2011).

Aumentar la eficiencia reproductiva es lo más importante para tener mayor aprovechamiento e intensificación del ritmo de mejora genética de los animales. A causa del fotoperiodo, la incidencia de ovulaciones varía durante el año, lo cual limita la reproducción de la yegua; por lo que tratar con hormonoterapia desempeña una función primordial (Ferris et al., 2012). Entre los beneficios de la hormonoterapia, están: el aumento del periodo de ciclicidad durante el año, el aumento del número de ovulaciones, la posibilidad de que el ambiente uterino propicie el desarrollo embrionario, la inducción de parto, la ayuda en el tratamiento de infecciones uterinas y la contribución en la utilización de biotécnicas de congelación de embriones, la fertilización in vitro y la vitrificación de embriones (Faria y Gradela, 2010).

La inducción de la ovulación se ha vuelto un método de rutina en reproducción equina, ya que la inseminación artificial y transferencia de embriones requieren una precisa predicción del tiempo de ovulación, lo cual avala el uso de fármacos (Cortés-Vidauri et al., 2018). La gonadotropina coriónica humana (hCG), ha sido la primera hormona utilizada para inducir la ovulación en yeguas y es la más utilizada; sin embargo, el uso frecuente de esta hormona produce la formación de anticuerpos anti-hCG, con una subsecuente pérdida de la eficacia farmacológica (Figuereido et al., 2011). El acetato de deslorelina (AD), es un fármaco creado como antagónico de la hormona liberadora de las gonadotropinas $(\mathrm{GnRH})$, y tiene como ventaja que su uso repetido no disminuye su eficacia (Ferris et al., 2012).

Los protocolos de manejo reproductivo con $\mathrm{GnRH}$ en yeguas anéstricas para inducir el estro y la ciclicidad, proporcionan evidencia de que hay ovulaciones múltiples en algunas hembras con tales regímenes de tratamiento. Al respecto, se ha reportado que aplicar acetato de deslorelina con al menos dos folículos con diámetros entre 20 a $24 \mathrm{~mm}$, puede producir doble ovulación en yeguas (Nagao et al., 2012).

Por lo anterior, el objetivo del presente estudio es evaluar la respuesta de los tratamientos hormonales con acetato de deslorelina y gonadotropina coriónica humana en la actividad ovárica en yeguas postparto Cuarto de Milla, con folículos ováricos $\geq 35 \mathrm{~mm}$ de diámetro. 


\section{MATERIAL Y MÉTODOS}

Localización del área de estudio. El estudio se realizó durante enero a junio de 2018 en el Rancho "Mogotes", localizado en Aldama, municipio de Irapuato, Guanajuato,

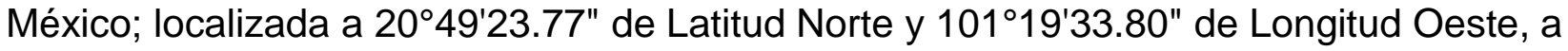
una altitud de $1700 \mathrm{msnm}$, con clima cálido subhúmedo, temperatura media anual de $20.5^{\circ} \mathrm{C}$ y precipitación pluvial de $692 \mathrm{~mm}$ (INAFED, 2018).

Animales experimentales y manejo. Se utilizaron dieciséis yeguas postparto de la raza Cuarto de Milla, clínicamente sanas, de 7 años, $450 \mathrm{~kg}$ de peso y 4.5 partos en promedio. Las yeguas se mantuvieron en condiciones de alimentación adecuada y con buena condición corporal, lo cual se requiere para el desempeño reproductivo apropiado; representado en ciclos estrales regulares y buena calidad de los folículos ováricos. El presente estudio se realizó de acuerdo con los estándares para el uso y cuidado de animales de investigación de acuerdo con las Normas Oficiales Mexicanas NOM-024ZOO-1995 y la NOM-051-ZOO-1995 (NOM-024- ZOO-1995; NOM-051-ZOO-1995).

Protocolo experimental y tratamientos. Se utilizó un diseño completo con tratamientos aleatorizados, en el cual las yeguas se asignaron al azar a uno de dos tratamientos ( $T$, figura 1): T1 ( $\mathrm{n}=8$ ): aplicación de $1 \mathrm{mg}$ de acetato de deslorelina (AD; Sincrorrelin®, Lab. Ourofino) por yegua con crecimiento folicular $\geq 35 \mathrm{~mm}$ de diámetro; y T2 $(n=8)$ : aplicación de $2500 \mathrm{UI}$ de gonadotropina coriónica humana liofilizada (hCG; CHORULON®, Lab. Merk Sharp \& Dohme), por cada yegua con crecimiento folicular $\geq 35 \mathrm{~mm}$ de diámetro. Los tratamientos hormonales se aplicaron vía intramuscular con jeringas de 3 y $5 \mathrm{~mL}$ (Terumo®) y aguja hipodérmica 21G $(32 \mathrm{~mm}$ ), previa desinfección de la zona de aplicación con torundas de algodón humedecidas con alcohol.

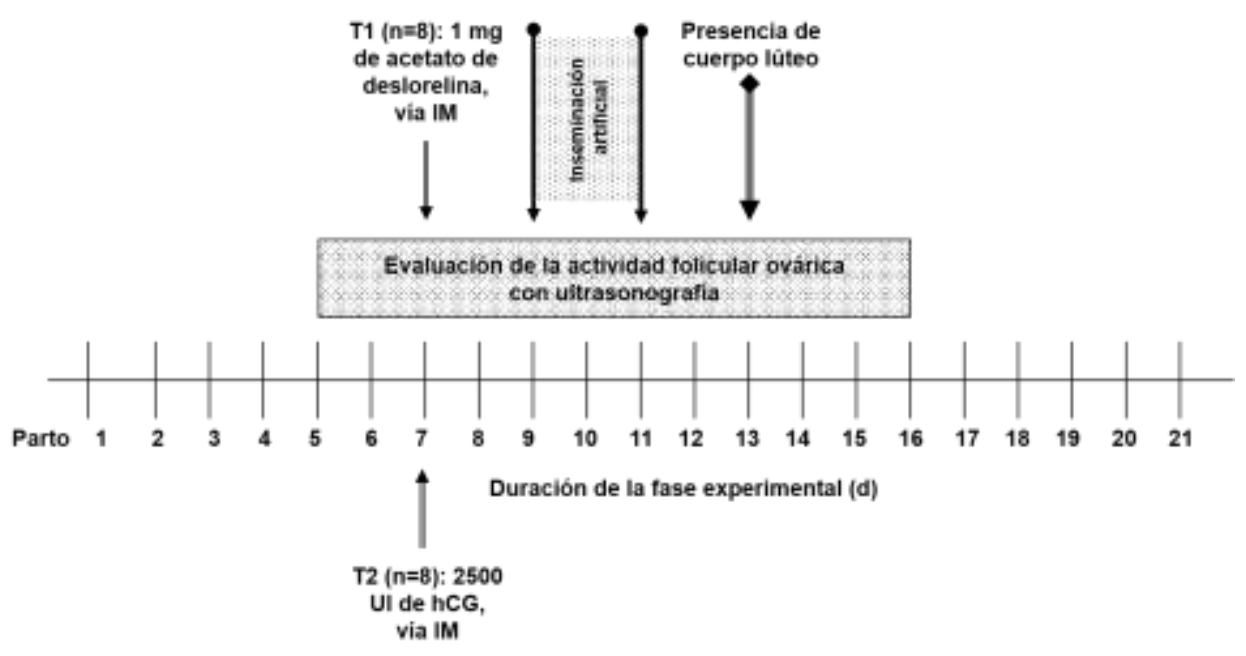

Figura 1. Protocolo de manejo reproductivo en yeguas Cuarto de Milla 
Evaluación de la actividad ovárica. Todas las yeguas se exploraron por ultrasonografía transrectal, a tiempo real con un transductor de 7.5 Mhz (CHISON ECO2, China); durante los 9 y 11 días postparto, conocido como "calor del potro", para conseguir la preñez se realizó servicio con semen refrigerado por inseminación artificial (IA). El diámetro folicular ovárico se evaluó cada $24 \mathrm{~h}$ desde el inicio del estro postparto, hasta que se detectó la ovulación mediante la imagen anecogénica en el ovario por la presencia del cuerpo hemorrágico, posterior a la IA. Se consideró como diámetro del folículo dominante, la medida registrada en el día previo a la aparición del cuerpo lúteo. Al observar folículos ováricos mayores o iguales a $35 \mathrm{~mm}$ de diámetro, se aplicaron los tratamientos hormonales T1: AD y hCG. Se determinó la tasa de crecimiento folicular ovárico $(\mathrm{mm})$, la respuesta a la ovulación (h) y el porcentaje de ovulación (\%).

Análisis estadístico. Los datos se analizaron con SAS (SAS Institute Inc, 2012). Para analizar los datos de la actividad ovárica, se realizó un análisis de varianza con medidas repetidas en el tiempo; se utilizó la prueba de Tukey para la comparación múltiple de medias $(P<0.05)$. Para analizar el tiempo de respuesta a la ovulación, se utilizó la prueba de log Rank con el procedimiento LIFETEST, mediante las curvas de supervivencia, y para evaluar la respuesta de ovulación (porcentaje) se utilizó la prueba de $\chi^{2}$.

\section{RESULTADOS Y DISCUSIÓN}

Desarrollo folicular ovárico. Las yeguas postparto Cuarto de Milla tratadas con AD y hCG presentaron desarrollo folicular ovárico similar $(P>0.05)$, evaluado durante la fase experimental (cuadro 1). La tasa de crecimiento folicular ovárico fue similar $(P>0.05)$ entre tratamientos (AD: $0.48 \pm 0.006$ y hCG: $0.45 \pm 0.035$ ). No se encontraron diferencias $(P>0.05)$ en el tiempo de respuesta a la ovulación (figura 2$)$ y en el porcentaje de ovulación (cuadro 2).

Cuadro 1. Desarrollo folicular ovárico en yeguas postparto Cuarto de Milla tratadas con acetato de deslorelina y gonadotropina coriónica humana

\begin{tabular}{|c|c|c|c|c|c|}
\hline \multirow{2}{*}{ TRATAMIENTO (T) } & \multicolumn{5}{|c|}{ Diámetro folicular (cm) } \\
\hline & Día 5 & Día 6 & Día 7 & Día 8 & Día 9 \\
\hline $\begin{array}{c}\text { T1 }(\mathrm{n}=8) \\
\text { Acetato de deslorelina }\end{array}$ & $2.55 \mathrm{a}$ & $3.05 \mathrm{a}$ & $3.53 \mathrm{a}$ & $4.00 \mathrm{a}$ & $4.48 \mathrm{a}$ \\
\hline $\begin{array}{l}\mathrm{T} 2(\mathrm{n}=8) \\
\text { Gonadotropina coriónica equina }\end{array}$ & $2.70 \mathrm{a}$ & $3.10 \mathrm{a}$ & $3.5 \mathrm{a}$ & $4.05 \mathrm{a}$ & $4.5 \mathrm{a}$ \\
\hline E.E.M. & 0.18 & 0.11 & 0.05 & 0.05 & 0.08 \\
\hline$P$-value & 0.4358 & 0.6704 & 0.6202 & 0.3559 & 0.7502 \\
\hline
\end{tabular}

a: Medias con literal similar en la misma columna son iguales $(P>0.05)$.

La administración de $1 \mathrm{mg}$ de acetato de deslorelina vía IM al momento de identificar un folículo dominante de 38 a $40 \mathrm{~mm}$ de diámetro, redujo el tiempo de ovulación entre 24 y 48 h y por lo tanto, favoreció el número de servicios por concepción, en comparación con la respuesta del tratamiento con gonadotropina coriónica humana utilizada en el presente 
estudio. Lo anterior se puede explicar debido a que los porcentajes de concepción y de ovulación son de $55 \%$ y $60 \%$ respectivamente, después de 14 a 40 horas de aplicar las hormonas (Figuereido et al., 2011). La ovulación en las yeguas Cuarto de Milla, en promedio ocurre con folículos de $40 \mathrm{~mm}$ de diámetro (Rodríguez et al., 2013). El momento óptimo para la aplicación de hCG, es cuando la yegua tiene un folículo dominante mayor o igual a $35 \mathrm{~mm}$ de diámetro, con una dosis de $3000 \mathrm{UI}$. La ovulación se presenta entre las 24 a 48 horas después de la aplicación (Dolezel et al., 2012).

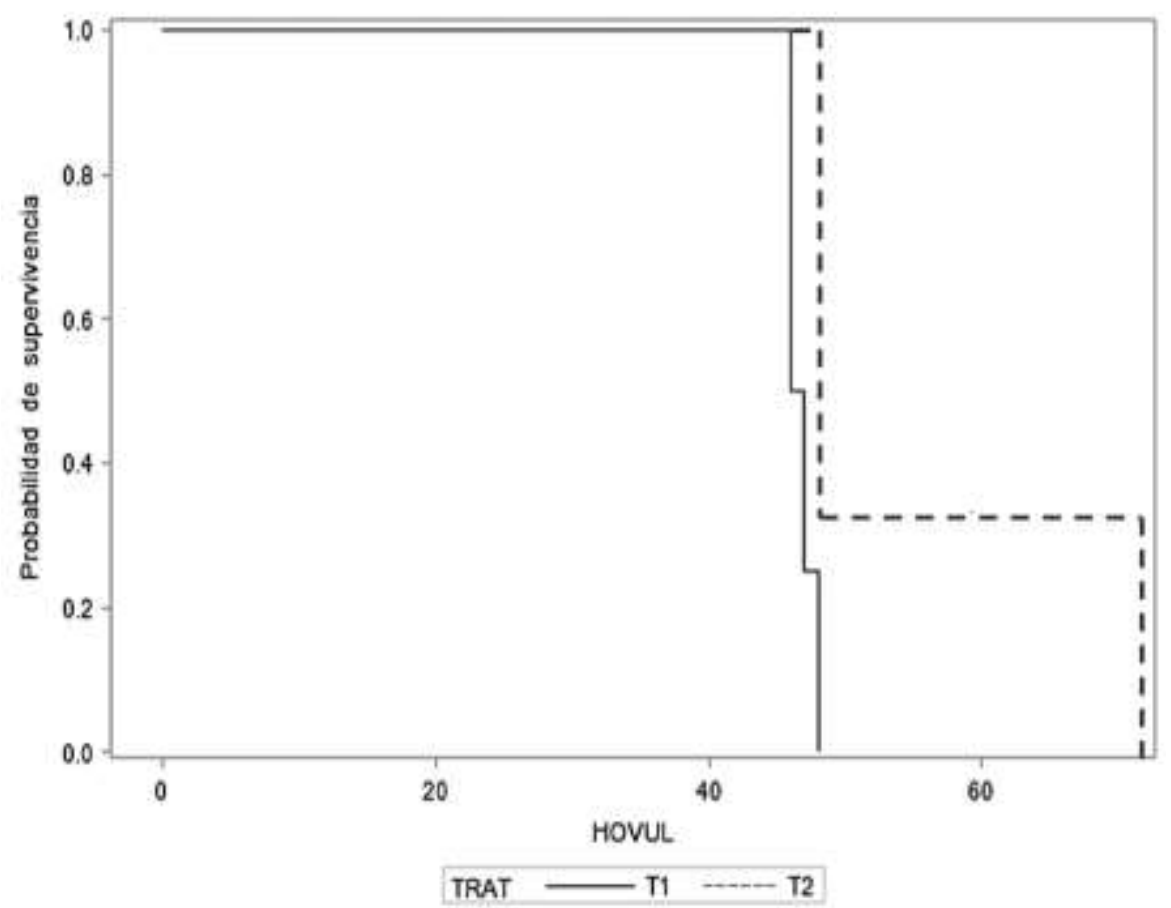

Figura 2. Curva de supervivencia para el tiempo de respuesta a la ovulación (h) en yeguas Cuarto de Milla, tratadas con acetato de deslorelina (T1) y gonadotropina coriónica humana (T2), HOVUL: Horas a la ovulación

Respuesta a la ovulación. No se encontraron diferencias $(P>0.05)$ en el tiempo de respuesta a la ovulación y en el porcentaje de ovulación (cuadro 2).

Cuadro 2. Respuesta a la ovulación en yeguas Cuarto de Milla, tratadas con acetato de deslorelina y gonadotropina coriónica humana

\begin{tabular}{cccc}
\hline Tratamiento $(\mathrm{T})$ & $\begin{array}{c}\text { Tiempo de respuesta a } \\
\text { la ovulación }(\mathrm{h})\end{array}$ & $\begin{array}{c}\text { Ovulación } \\
(\%)\end{array}$ & $\begin{array}{c}\text { Ovulación } \\
\text { por ovario derecho } \\
(\%)\end{array}$ \\
\hline $\begin{array}{c}(\mathrm{T} 1, \mathrm{n}=8) \\
\begin{array}{c}\text { Acetato de deslorelina } \\
(\mathrm{T} 2, \mathrm{n}=8)\end{array}\end{array}$ & $46.75 \pm 0.48 \mathrm{a}$ & $8 / 8(100 \%) \mathrm{a}$ & $75.0 \mathrm{a}$ \\
$\begin{array}{c}\text { Gonadotropina coriónica } \\
\text { humana }\end{array}$ & $56.00 \pm 8.00 \mathrm{a}$ & $6 / 8(87.5 \%) \mathrm{a}$ & $75.0 \mathrm{a}$ \\
\hline a: Medias con literal similar en la misma columna son iguales $(P>0.05)$. &
\end{tabular}


Los resultados del presente estudio coinciden con los reportados por Dolezel et al. (2012), quienes administraron vía intravenosa $3000 \mathrm{UI}$ de hCG durante el estro en yeguas con un folículo dominante $\geq 35 \mathrm{~mm}$ de diámetro, y observaron que las ovulaciones ocurrieron entre 12 y $48 \mathrm{~h}$ después del tratamiento. Al respecto, es posible que el crecimiento del folículo dominante después de aplicar la dosis de hCG, haya sido influenciado por el tamaño de los folículos en el momento del tratamiento. Finan et al. (2016) encontraron que el $93.75 \%$ de las yeguas ovularon dentro de las $48 \mathrm{~h}$ después del tratamiento con deslorelina vía IM; a diferencia de Chávez et al. (2018), quienes reportaron que el tiempo de ovulación fue de $43.13 \pm 4.48 \mathrm{~h}$ y $69.00 \pm 8.41 \mathrm{~h}$ para las yeguas tratadas con acetato de deslorelina y el tratamiento testigo, respectivamente. El $87.5 \%(7 / 8)$ de las yeguas tratadas con acetato de deslorelina ovularon dentro de las $48 \mathrm{~h}$; mientras que sólo el $37.5 \%(3 / 8)$ para las del tratamiento testigo.

Las diferencias observadas entre los estudios podrían deberse al tamaño de muestra experimental, o a un factor ambiental, nutricional o de raza (Boeta et al., 2006). La capacidad de inducir la ovulación en un momento predecible, ha aumentado la eficiencia de los programas de reproducción equina, donde los beneficios incluyen una disminución en el número de servicios por ciclo para sementales; lo que permite mayores registros de yeguas anualmente (Finan et al., 2016).

Los protocolos de manejo reproductivo equino, estimulan la inducción de la ovulación para proporcionar una ovulación programada en yeguas, para un manejo óptimo de la reproducción. Al respecto, se han reportado numerosos estudios sobre la eficacia de prostaglandinas, $\mathrm{GnRH}$, análogos de $\mathrm{GnRH}$ y hCG para inducir la ovulación en la yegua (Yoon, 2012). Se ha reportado que el diámetro, la forma fluctuación del folículo dominante y el plegamiento endometrial, se pueden considerar para estimar la predicción de la ovulación; sin embargo, no son lo suficientemente precisos ni estándar (Ramírez et al., 2010). Por lo tanto, es importante la inducción o sincronización hormonal de la ovulación, la cual favorece la determinación del momento óptimo para la inseminación. La administración de gonadotropina coriónica humana $(\mathrm{hCG})$, representa el método de tratamiento más frecuente para este propósito (Dolezel et al., 2012). Por el contrario, la anovulación es una actividad fisiológica normal en la yegua durante la transición de la primavera al otoño; sin embargo, el desarrollo folicular anovulatorio ocurre ocasionalmente en la época reproductiva. Lo anterior, puede explicarse debido a que los folículos crecen entre 5 y $15 \mathrm{~cm}$ de diámetro y persisten hasta dos meses. Estos folículos producen comportamiento de estro anormal y prolongan el periodo interovulatorio (Ángel y Bran, 2010). 


\section{CONCLUSIÓN}

Los tratamientos hormonales con acetato de deslorelina y gonadotropina coriónica humana, responden en la actividad ovárica en las yeguas postparto Cuarto de Milla con folículos ováricos $\geq 35 \mathrm{~mm}$ de diámetro; por lo tanto, los dos tratamientos hormonales son opciones viables para controlar el desarrollo folicular ovárico, e inducir la ovulación en yeguas postparto Cuarto de Milla en protocolos de manejo reproductivo.

\section{LITERATURA CITADA}

ÁNGEL D, Bran JA. 2010. Reproducción asistida en equinos: nuevos aportes desde la teoría. Revista CES Medicina Veterinaria y Zootecnia. 5: 56-69. ISSN: 1900-9607. http://revistas.ces.edu.co/index.php/mvz/article/viewFile/984/1920

AURICH C. 2011. Reproductive cycles of horses. Animal Reproduction Science. 124(34): 220-228. ISSN: 0378-4320. https://doi.org/10.1016/j.anireprosci.2011.02.005

BOETA M, Porras A, Zarco LA, Aguirre-Hernández, R. 2006. Ovarian activity of the mare during winter and spring at a latitude of $19^{\circ} 21^{\prime}$ North. Journal Equine Veterinary Science. 26: 55-58. ISSN: 0737-0806. https://doi.org/10.1016/j.jevs.2005.12.003

CHÁVEZ CE, Baltodano TJ, Caballero LC. 2018. Efecto del uso de acetato de deslorelina en la inducción de ovulación de yeguas Caballo Peruano de Paso. Revista de Investigaciones Veterinarias del Perú. 29(2): 713-719. ISSN: 1609-9117. http://dx.doi.org/10.15381/rivep.v29i2.14487

CORTÉS-VIDAURI Z, Aréchiga-Flores C, Rincón-Delgado M, Rochín-Berumen F, LópezCarlos M, Flores-Flores G. 2018. Revisión: El Ciclo Reproductivo de la Yegua. Abanico Veterinario. 8(3):14-41. ISSN: 2448-6132. http://dx.doi.org/10.21929/abavet2018.83.1

DOLEZEL R, Ruzickova K, Maceckova G. 2012. Growth of the dominant follicle and endometrial folding after administration of hCG in mares during oestrus. Veterinarni Medicina. 57(1): 36-41. https://www.agriculturejournals.cz/publicFiles/57597.pdf

FARIA DR, Gradela A. 2010. Hormonioterapia aplicada à ginecologia equina. Revista Brasileira de Reprodução Animal. 34(2): 114-22. https://www.researchgate.net/publication/285119665_Hormonioterapia_aplicada_a_gine cologia_equina

FERRIS RA, DACT, Hatzel JN, Lindholm ARG, Scofield DB, McCue PM. 2012. Efficacy of deslorelin acetate (sucromate) on induction of ovulation in American Quarter Horse mares. Journal of Equine Veterinary Science. 32 (5): 285-288. ISSN: 0737-0806, https://doi.org/10.1016/j.jevs.2011.11.007 
FINAN SA, Lamkin EL, McKinon AO. 2016. Comparative efficacy of BioRelease Deslorelin® injection for induction of ovulation in oestrus mares: a field study. Australian Veterinary Journal. 94: 338-340. ISSN: 1751-0813. https://doi.org/10.1111/avj.12478

FIGUEIREDO T, Paiva R, Kozicki LE, Kaercher F, Weiss RR, Dos Santos IW, Muradas P. 2011. Induction of ovulation in quarter horse mares through the use of deslorelin acetate and human chorionic gonadotrophin (hCG). Brazilian Archives of Biology and Technology. 54: 517-521. ISSN: 1516-8913. https://doi.org/10.1590/S151689132011000300012

INAFED, Instituto Nacional para el Federalismo y el Desarrollo Municipal. 2018. http://www.inafed.gob.mx/work/enciclopedia/EMM11guanajuato/municipios/11017a.html

NAGAO JF, Neves Neto JR, Papa FO, Alvarenga MA, Freitas-Dell'Aqua CP, Dell'Aqua Junior JA. 2012. Induction of double ovulation in mares using deslorelin acetate. Animal Reproduction Science. 136: 69-73. https://doi.org/10.1016/j.anireprosci.2012.10.015

NORMA Oficial Mexicana. NOM-024-ZOO-1995. Especificaciones y características zoosanitarias para el transporte de animales, sus productos y subproductos, productos químicos, farmacéuticos, biológicos y alimenticios para uso en animales o consumo por éstos. https://www.gob.mx/cms/uploads/attachment/file/202301/NOM-024-ZOO1995_161095.pdf

NORMA Oficial Mexicana. NOM-051-ZOO-1995. Trato humanitario en la movilización de animales. https://www.gob.mx/cms/uploads/attachment/file/203479/NOM-051-ZOO1995_230398.pdf

RAMíREZ G, Gutiérrez C, Ramos M. 2010. Dinámica folicular en yeguas Paso Fino Colombiano medido por ultrasonografía en la Sabana de Bogotá. Revista de Medicina Veterinaria. 19: 21-35. http://www.scielo.org.co/pdf/rmv/n19/n19a03.pdf

RODRÍGUEZ GA, Bazán GA, Rodríguez GJ, Espinoza BJ, Vásquez CM, Lucas LJ, Huanca LW. 2013. Evaluación del folículo ovárico de yeguas criollas postadministración de hCG. Revista de Investigaciones Veterinarias del Perú. 24(2): 189-193. ISSN: 1609$9117 . \quad$ http://www.scielo.org.pe/scielo.php?script=sci_arttext\&pid=S160991172013000200008

SAS Institute. 2012. Statistical Analysis Software SAS/STAT®. version 9.0.2, Cary, N.C., USA: SAS Institute Inc., ISBN: 978-1-60764-599-3. http://www.sas.com/en_us/software/analytics/stat.html\#

YOON M. 2012. The estrous cycle and induction of ovulation in mares. Journal of Animal Science and Technology. 54(3): 165-174. http://dx.doi.org/10.5187/JAST.2012.54.3.165 